\title{
Can type II Semi-local cosmic strings form?
}

\author{
Betti Hartmann* \\ School of Engineering and Science, Jacobs University Bremen, 28759 Bremen, Germany \\ Patrick Peter \\ $G \mathbb{R} \varepsilon \mathbb{C O}$ - Institut d'Astrophysique de Paris, UMR7095 CNRS, \\ Université Pierre $\mathcal{E}$ Marie Curie, 98 bis boulevard Arago, 75014 Paris, France
}

(Dated: November 13, 2018)

\begin{abstract}
We present the simplest possible model for a semi-local string defect in which a U(1) gauged subgroup of an otherwise global $\mathrm{SU}(2)$ is broken to produce local cosmic strings endowed with current-carrying properties. Restricting attention to type II vortices for which the non current-carrying state is unstable, we show that a condensate must form microscopically and macroscopically evolve towards a chiral configuration. It has been suggested that such configurations could potentially exist in a stable state, thereby inducing large cosmological consequences based on equilibrium angular momentum supported loop configurations (vortons). Here we show that the current itself induces a macroscopic (longitudinal) instability: we conclude that type II semi-local cosmic strings cannot form in a cosmological context.
\end{abstract}

PACS numbers: 97.60.Jd,26.20.+c,47.75.+f,95.30.Sf

\section{INTRODUCTION}

Cosmic strings have long lost their status of plausible competitors to the inflation paradigm [1]. However, from the point of view of particle physics and high energy models thereof, the opposite should be true: even though it is not immediately obvious to build consistent models of inflation based on the most natural extensions of the standard model such as supersymmetric Grand Unified Theories (GUT) or strings, those naturally predict vortex-like objects, i.e. linear topological defects [2] (see however Ref. [3]). Thus, constraints provided by cosmic string network simulations are very much still of current interest, would it be only to understand why and how one can construct an inflation model without strings.

Assuming strings to form however is not yet the end of the story. In practice, most research has been made under the assumption that the vortices were not endowed with any particular structure, and hence that the spacelike twodimensional worldsheet they described was well modeled by a Nambu-Goto Lorentz invariant action, i.e. the area spanned by the worldsheet.

That such a model attracted attention makes full sense since it turns out that any more complicated model would be essentially intractable by means of the currently available technology. Besides, it was also shown that any Lorentz symmetry-breaking current on the vortices could lead to centrifugally-supported equilibrium states, dubbed vortons [4, 5], whose existence merely rules out the string scenario altogether [6], provided they are sufficiently long-lived.

*Electronic address: b.hartmann@jacobs-university.de

${ }^{\dagger}$ Electronic address: peter@iap.fr
Structureless Nambu-Goto strings, on the other hand, are very difficult to produce in almost any reasonable high energy theory. Indeed, and unless one assumes a special sector put by hand to generate the strings themselves, which comes very short of the original idea to describe the high energy phenomena in a unified and consistent way, the string-forming Higgs field present in most GUT model must couple to scalars, fermions or gauge fields in such a way as to produce currents. Even the cosmic strings present in the superstring framework do not escape this conclusion, as they must couple to moduli, at least the volume of the compact extra dimensions. Thus, one expects cosmic strings to be of the current-carrying kind, as originally introduced by Witten in 1985 [7].

Many models have since been discussed and investigated by numerous authors, with the general conclusion that the equation of state of the strings is highly non trivial, with specific properties such as the existence of a maximal spacelike current, a phase frequency threshold for timelike current above which there is no bound state anymore, and the possibility, in all known models, to build a lightlike current which ought to be absolutely stable, thus enhancing the vorton excess problem [8-10]. Solutions have been proposed, most of them based on the instabilities of current-carrying loop configurations that would dissipate most of the large loops before they have time to evolve into cosmologically dangerous vortons. The present work, although not directly concerned with this problem, suggests yet another possibility, namely that the current could form directly in a configuration that would be unstable with respect to longitudinal (soundlike along the string) modes.

Our model can be seen as the next-to-simple one after the neutral Witten bosonic model, consisting of a global $\mathrm{U}(1)$ condensate in a local $\mathrm{U}(1)$ vortex. Here we still 
assume the vortex to be produced by a gauged U(1) symmetry breaking, but instead of adding extra symmetries, we embed this local U(1) into an otherwise global SU(2). Non-current carrying strings in this model have been investigated in [11-14], while the current-carrying case has been discussed in [15, 16]. This is merely the limit of the usual would-be semi-local strings found in the standard electroweak model; except that the measured parameters of this model preclude their actual stability. In fact, the stability of non-current carrying semi-local strings does not follow from the topology of the vacuum manifold (as it does for the U(1) case), but from dynamical arguments.

The ratio between the gauge and Higgs boson masses governs the stability of semi-local strings: for Higgs boson mass larger (smaller) than the gauge boson mass semi-local strings are unstable (stable) and in the BPS limit a degenerate one-parameter family of stable solutions exists [12]. The parameter corresponds roughly to the width of the strings and as such semi-local strings of arbitrary width have the same energy in the BPS limit. Whenever this zero mode gets excited it leads to the growth of the string core [17]. As such these non-current carrying semi-local strings have been studied in the context of cosmological applications regarding the formation and evolution of string networks [18--21] as well as implications for the CMB [22]. The stability of the current-carrying counterparts has been discussed in [23] using linear perturbation theory; there it was also found that these embedded type II vortices have a single unstable mode, and so it has been suggested that the currentcarrying ones, being less energetic, could be stable. We show that this is not the case because some other instability develops.

In a sense, the category of this model is more natural than the Witten-kind of models because one expects a large GUT group to be partially broken to yield the low energy particle physics currently tested at the LHC, so the strings, if present, once formed, are expected to be embedded in a larger structure. It is obviously mostly a parameter dependent question to know whether the strings here described will form rather than the Witten kind of strings. Finally, such a model permits to embed a cosmic string in a non abelian framework in a tractable way, contrary to what happens in the case of a pure non abelian current-carrying situation [24, 25].

As already mentioned above, if the ratio between the Higgs and gauge boson masses is large, the corresponding type II vortices are unstable. In Ref. [15] and [16], it was shown that a current could build along such vortices, and that the resulting current-carrying state was less energetic than the structureless one. A stability analysis [23] then showed that even though long wavelength perturbations tend to grow exponentially, there was a limit below which the current-carrying string state could be stable; this could imply important cosmological consequences whenever small loops form. The purpose of the current article is to close this window of stability by performing a global analysis showing the current-carrying configurations will also develop a short wavelength instability, the so-called longitudinal instability introduced by Carter [26-28].

The paper is organized as follows: in the following section [I] we set up the actual model and discuss the stringlike solutions that can be expected. We then move on, in Sec. III to evaluating the currents that could condense in a string core, summarizing a stability analysis first discussed in [12]. These currents are examined thoroughly in Sec. IIIB and it is shown that the lightlike current limit is defined as the endpoint of the state parameter space in this case, with the phase frequency threshold being at the null point. Finally, Sec. [V] shows that the corresponding equation of state leads to the longitudinal loop instabilities: right after a condensate has formed, it should evolve towards the chiral limit [29], thereby destroying many would-be vortons [30] through emission of high energy particles [31, 32]. We conclude that type II vortices cannot form at all in such models.

\section{PARTLY GAUGED SU(2) STRING MODEL}

The simplest embedded current-carrying string model is provided by the partly ungauged version of the electroweak theory in which the SU(2) coupling constant is made to vanish, while the equivalent to electromagnetism $\mathrm{U}(1)$ remains gauged. In practice, this amounts to starting with the following Lagrangian

$$
\mathcal{L}=-g^{\mu \nu}\left(D_{\mu} \boldsymbol{\Phi}\right)^{\dagger} \cdot D_{\nu} \boldsymbol{\Phi}-\frac{1}{4} F_{\mu \nu} F^{\mu \nu}-V(\boldsymbol{\Phi}),
$$

where the $\mathrm{U}(1)$ covariant derivative acting on the $\mathrm{SU}(2)$ Higgs doublet $\boldsymbol{\Phi}$ is $D_{\mu} \boldsymbol{\Phi} \equiv\left(\partial_{\mu}-i e A_{\mu}\right) \boldsymbol{\Phi}, F_{\mu \nu} \equiv \partial_{\mu} A_{\nu}-$ $\partial_{v} A_{\mu}$ is the Faraday tensor of the U(1) gauge field, and finally the scalar field potential $V$ is taken to be of the symmetry-breaking kind

$$
V(\boldsymbol{\Phi})=\frac{\lambda}{2}\left(\boldsymbol{\Phi}^{\dagger} \cdot \boldsymbol{\Phi}-\eta^{2}\right)^{2},
$$

so the self coupling $\lambda$ combines with the vacuum expectation value (vev) $\eta$ of $\boldsymbol{\Phi}$ to provide the scalar field excitation mass as $m_{\phi}=\sqrt{2 \lambda} \eta$. The vector field also acquires a mass $m_{A}=\sqrt{2} e \eta$, and the mass ratio is thus defined as $2 \beta \equiv m_{\phi}^{2} / m_{A}=\lambda / e^{2}$. (Note our definition of $\beta$ differs by a factor of 2 with that of Ref. [15].)

The lowest energy configuration, having $\boldsymbol{\Phi}^{\dagger} \cdot \boldsymbol{\Phi}=\eta^{2}$ admits vortex defects of the local U(1) kind: fixing the $\mathrm{SU}(2)$ gauge in which

$$
\boldsymbol{\Phi}_{0}=\left(\begin{array}{c}
\Phi_{0} \\
0
\end{array}\right)
$$


there remains a local $U(1)$ gauge to be fixed through the phase of $\Phi_{0}$; if it takes the form of a non vanishing winding, i.e. if $\Phi_{0} \propto \mathrm{e}^{i n \theta}$ with index $n \in \mathbb{Z} \neq 0$ and $\theta$ a local coordinate angle, then $\Phi_{0} \rightarrow 0$ defines a string around which the phase winds. One can then locally set the string to be aligned along a $z$-axis around which one defines the cylindrical coordinates $r$ and $\theta$, and the non vanishing component of the Higgs field becomes $\Phi_{0}=\varphi(r) \mathrm{e}^{i n \theta}$, where $\lim _{r \rightarrow \infty} \varphi(r)=\eta$ and $\varphi(0)=0$.

The question then arises as to the actual stability of the above configuration. An analysis similar to that in [7] is carried out below showing that one does indeed expect a current of the kind we discussed in the following sections.

From the Lagrangian (1), one obtains the general equations of motion for the gauge field $A_{\mu}$ as

$$
\frac{1}{\sqrt{-g}} \partial_{\nu}\left(\sqrt{-g} F^{\nu \mu}\right)=2 e^{2} \boldsymbol{\Phi}^{\dagger} \cdot \boldsymbol{\Phi} A^{\mu}+i e \boldsymbol{\Phi} \stackrel{\leftrightarrow}{\partial^{\mu}} \boldsymbol{\Phi},
$$

and for the Higgs scalar

$$
\frac{1}{\sqrt{-g}} \partial_{\mu}\left(\sqrt{-g} g^{\mu v} D_{\nu} \boldsymbol{\Phi}\right)=i e A^{v} D_{\nu} \boldsymbol{\Phi}+\boldsymbol{\Phi} \frac{\mathrm{d} V(\boldsymbol{\Phi})}{\mathrm{d}\left(\boldsymbol{\Phi}^{\dagger} \cdot \boldsymbol{\Phi}\right)},
$$

with the hermitian conjugate equation applying for $\boldsymbol{\Phi}^{\dagger}$. These give, for the background configuration (3) with the potential [2],

$$
\frac{\mathrm{d}^{2} \varphi}{\mathrm{d} r^{2}}+\frac{1}{r} \frac{\mathrm{d} \varphi}{\mathrm{d} r}=\left[\frac{Q^{2}}{r^{2}}+\lambda\left(\varphi^{2}-\eta^{2}\right)\right] \varphi
$$

and

$$
\frac{\mathrm{d}^{2} Q}{\mathrm{~d} r^{2}}-\frac{1}{r} \frac{\mathrm{d} Q}{\mathrm{~d} r}=2 e^{2} \varphi^{2} Q
$$

after setting $Q=n-e A_{\theta}$ to account for the winding number. We now assume - see the following sections that we have (numerical) solutions for the functions $\varphi(r)$ and $Q(r)$.

Because the Higgs doublet is coupled with itself, and even though finite energy solutions of Eqs. (6) and (7) exist, one needs verify that these are stable. Following Witten [7], we set an arbitrary perturbation $\boldsymbol{\Phi}=\boldsymbol{\Phi}_{0}+\delta \boldsymbol{\Phi}$ with

$$
\delta \boldsymbol{\Phi}=\left(\begin{array}{c}
0 \\
\sigma \mathrm{e}^{i \omega t}
\end{array}\right),
$$

where $\sigma=\sigma(r)$ depends on the radial coordinate only. Plugging Eq. (11) into (5) and keeping only first order terms, one gets the Schrödinger-like equation

$$
-\Delta_{2} \sigma+\mathcal{V}(r) \sigma=\omega^{2} \sigma,
$$

where $\Delta_{2}=\partial_{x}^{2}+\partial_{y}^{2}=\partial_{r}^{2}+r^{-1} \partial_{r}+r^{-2} \partial_{\theta}^{2}$ is the twodimensional laplacian and the effective potential $\mathcal{V}$ reads

$$
\mathcal{V}(r)=\frac{[n-Q(r)]^{2}}{r^{2}}+\lambda\left[\varphi^{2}(r)-\eta^{2}\right] .
$$

This potential is shown on Fig. 1 for different values of the parameter $\beta \equiv \lambda /\left(2 e^{2}\right)$. One expects from the figure that there could be bound states provided $\beta$ is large enough.

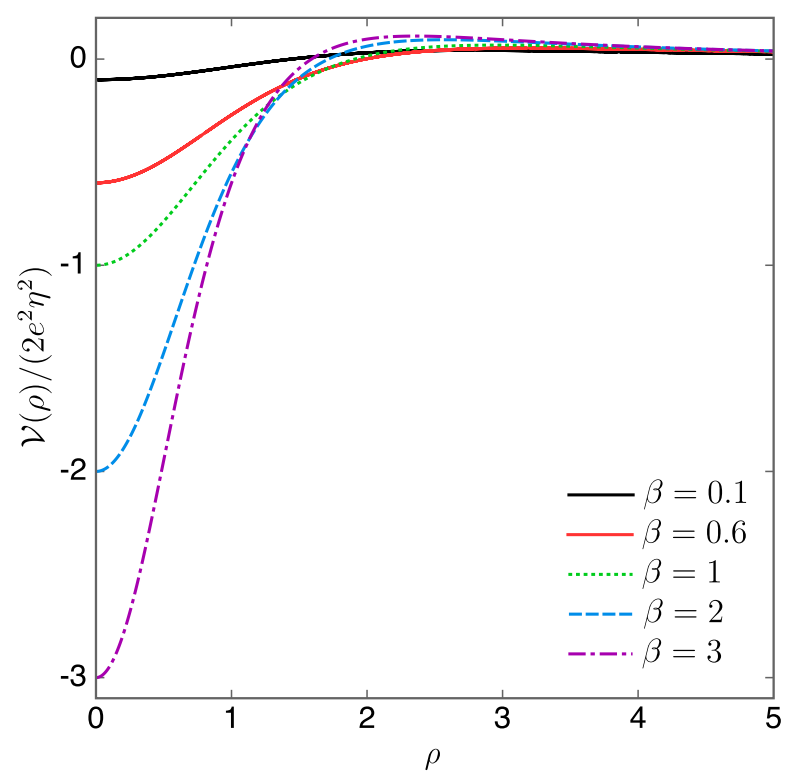

FIG. 1: The potential [10, rescaled so as to be dimensionless, appearing in the Schrödinger equation 9 for various values of the parameter $\beta=\lambda /\left(2 e^{2}\right)$ as a function of the dimensionless distance to the string core $\rho=\sqrt{2} e \eta r$ (see Sec. IV C for details on the numerics).

Since $\lim _{r \rightarrow 0} \mathcal{V}(r)=-\lambda \eta^{2}$ is negative and $\mathcal{V} \sim n^{2} / r^{2}$ asymptotically, the potential satisfies the usual quantum mechanical conditions for having a bound state: a range of values for the parameter $\beta$ can be found for which there exist solutions of Eq. 9 with $\omega^{2}<0$, and hence an instability of the background solution (3) should develop. With the non linear terms taken fully into account, the instability translates into a condensate that can carry a current. Comparison with Ref. [12] shows that for $\beta>\frac{1}{2}$, i.e. $\lambda>e^{2}$, one expects a condensate to form: according to the usual classification, this means that type I vortices are absolutely stable (no condensate) while type II vortices spontaneously form a current-carrying state. Note also that since type II vortices are energetically favored to occur with unit winding number, we shall for now on restrict attention to the case $n=1$. The question now is whether or not these current-carrying solutions can lead to the stable enough configurations (for cosmological purposes) discussed in [23].

It should be remarked at this stage that the mere existence of an instability does not guarantee that it has an endpoint which one then identifies with the currentcarrying state. The numerical solutions obtained below show that it does, and because the field equations stem from minimizing the energy per unit length to be mini- 
mized, they provide more stable configurations satisfying the boundary conditions. As we shall see, these solutions will turn out to initiate another instability.

\section{THE CURRENT-CARRIER CONDENSATE}

For now on, we follow [23] and assume a condensate did form and we write the Higgs doublet as

$$
\boldsymbol{\Phi}=\left[\begin{array}{l}
\varphi(r) \mathrm{e}^{i n \theta+i \psi(z, t)} \\
\sigma(r) \mathrm{e}^{i m \theta+i \xi(z, t)}
\end{array}\right],
$$

where $n \in \mathbb{Z}$ is the winding number of the string, $m \in \mathbb{Z}$ leaves the possibility for the perturbation to wind as well, and the phases $\psi$ and $\xi$ only depend on the internal string coordinates. This field can then source $A_{\theta}, A_{z}$ and $A_{t}$, all being functions of the radius $r$ only in order for the worldsheet to be localized. Note that the form (11) assumes no modes are present in the transverse direction, i.e. the phases $\psi$ and $\xi$ do not depend on $r$, so we do consider neither ingoing nor outgoing waves: the field configuration we are investigating is at equilibrium, hence may only have excitations along the worldsheet. We shall also occasionally use a latin index to denote worldsheet coordinates $\{z, t\}$ collectively.

\section{A. State parameters}

With the ansatz (11), the field equations now read

$$
A_{a}^{\prime \prime}+\frac{1}{r} A_{a}^{\prime}+2 e\left[\left(\partial_{a} \psi-e A_{a}\right) \varphi^{2}+\left(\partial_{a} \xi-e A_{a}\right) \sigma^{2}\right]=0,
$$

for the internal gauge fields,

$$
Q^{\prime \prime}-\frac{1}{r} Q^{\prime}=2 e^{2}\left[Q \varphi^{2}+(Q+m-n) \sigma^{2}\right],
$$

with the same convention as before that $Q=n-e A_{\theta}$,

$$
\varphi^{\prime \prime}+\frac{1}{r} \varphi^{\prime}=\left[P_{\psi}^{2}+\frac{Q^{2}}{r^{2}}+\lambda\left(\varphi^{2}+\sigma^{2}-\eta^{2}\right)\right] \varphi,
$$

with $P_{\psi}^{2}=\left(\partial_{z} \psi-e A_{z}\right)^{2}-\left(\partial_{t} \psi-e A_{t}\right)^{2}$,

$$
\sigma^{\prime \prime}+\frac{1}{r} \sigma^{\prime}=\left[P_{\xi}^{2}+\frac{(Q+m-n)^{2}}{r^{2}}+\lambda\left(\varphi^{2}+\sigma^{2}-\eta^{2}\right)\right] \sigma,
$$

where $P_{\xi}^{2}$ is defined in a similar fashion as $P_{\psi}$, namely $P_{\xi}^{2}=\left(\partial_{z} \xi-e A_{z}\right)^{2}-\left(\partial_{t} \xi-e A_{t}\right)^{2}$. Finally, the phases represent massless modes propagating along the string, as is clear from their equations of motion

$$
\left(\partial_{t}^{2}-\partial_{z}^{2}\right) \psi=\gamma^{a b} \partial_{a} \partial_{b} \psi=0=\gamma^{a b} \partial_{a} \partial_{b} \xi .
$$

In Eqs. (12) to (16, we have set a prime to denote a derivative with respect to the radial distance $r$.
One now needs to look at the boundary conditions to restrict attention to the physically meaningful cases. In particular, noting that $\lim _{r \rightarrow 0} Q(r)=n$ and assuming $P_{\xi}^{2}$ to be regular at the string core location, Eq. (15) implies the following: setting $\sigma \simeq \sigma_{0}+\sigma_{0}^{\prime} r+\frac{1}{2} \sigma_{0}^{\prime \prime} r^{2}+\cdots$, the expansion

$\sigma_{0}^{\prime \prime}\left(2-\frac{m^{2}}{2}\right)+\frac{\sigma_{0}^{\prime}}{r}\left(1-m^{2}\right)+\frac{m^{2} \sigma_{0}}{r^{2}} P_{\xi}^{2}(0) \sigma_{0}+O(r)=0$

should hold. In order for the $r^{-2}$ term to be regular, one must impose either $m=0$ or demand that $\sigma_{0}=0$. In the latter case, assuming $m \neq 0$, one finds that $m^{2}=1$ and $m^{2}=4$ simultaneously, which is self-contradictory. Hence, we must set $m=0$ and $\lim _{r \rightarrow 0} \sigma^{\prime}(r)=0$. Moreover, asymptotically, i.e. when $Q \rightarrow 0, \sigma \rightarrow 0$ and $\varphi \rightarrow \eta$, Eq. (12) becomes

$$
A_{a}^{\prime \prime}+\frac{1}{r} A_{a}^{\prime}+2 e \eta^{2}\left(\partial_{a} \psi-e A_{a}\right)=0
$$

the solution of which can only be made to vanish - i.e. we demand $\lim _{r \rightarrow \infty} A_{a}(r)=0$ in order for the total energy of the configuration to be finite - only provided $\partial_{a} \psi=0$. As $\psi$ must now be a constant, it can, without lacking generality, be set to zero by means of a global SU(2) gauge transformation (which can also remove any constant part that would be present in $\xi$ as well). The general solution of 16 then reads

$$
\xi=\xi_{-}(z-t)+\xi_{+}(z+t)+k z-\omega t,
$$

where $\xi_{ \pm}$represent the left and right massless modes moving along the string and the last term represents a coherent mode, that can, in the usual case, be built as a superposition of left and right movers. If a string segment is considered, the left and right moving modes are responsible for the leaking out of the current; again, following [23], we shall in what follows consider a $z$-independent string (approximating a closed loop when setting periodic boundary conditions), assuming it can somehow be formed in the first place and thus neglect these modes; we shall accordingly set $\xi_{ \pm} \rightarrow 0$ in what follows.

Because of Eq. (18), the last term of Eq. (17) is a constant. This implies that the two functions $P_{a} \equiv e A_{a}-\partial_{a} \xi$ satisfy the same linear equation and hence are merely proportional to one another for all values of $r$. One then has $P_{z} \propto P_{t}$, the proportionality constant being found by taking the asymptotic limit of this relation for which we want the gauge field $A_{a}$ to vanish. This yields $P_{z}=-k P_{t} / \omega$, and thus $A_{z}=-k A_{t} / \omega$. We are now in a position to define the relevant degree of freedom as

$$
A_{z}^{2}-A_{t}^{2}=\left(\frac{k^{2}}{\omega^{2}}-1\right) A_{t}^{2}=\left(1-\frac{\omega^{2}}{k^{2}}\right) A_{z}^{2} \equiv w P^{2},
$$

with $w$ the state parameter, and the function $P$ is dimensionless. The fields $A_{z}$ and $A_{t}$ are then related to $P$ 
through

$$
A_{t}=\omega P \sqrt{\frac{w}{k^{2}-\omega^{2}}}, \quad \text { and } \quad A_{z}=-k P \sqrt{\frac{w}{k^{2}-\omega^{2}}} ;
$$

note that $w$ has dimensions of a squared mass. In view of this, one needs to complement the system with yet another independent - and dimensionless - parameter $b$, representing the bias between the gauge fields and the phase gradient, through

$$
k^{2}-\omega^{2}=w b^{2} .
$$

The sign of $w$ determines that of the phase gradient, so the current is described by two positive parameters and a sign. For $w>0$ (resp. $w<0$ ), the current is spacelike (resp. timelike), and the equation of motion for $P$ is

$$
P^{\prime \prime}+\frac{1}{r} P^{\prime}=2 e^{2} P\left(\varphi^{2}+\sigma^{2}\right)+2 e b \sigma^{2},
$$

where we assume $b>0$.

Having constructed the current-carrying configuration and taken account of all the symmetries, we now turn to the range of parameters that one should investigate to fully describe such strings.

\section{B. The lightlike current limit}

The ordinary - neutral [33] or charged [34] - currentcarrying cosmic string is known to have a maximum charge density (timelike current) above which it is energetically favored for the condensed particles to form ingoing and outgoing massive radial modes. In the model here discussed, such a phase frequency threshold is also acting, and as it turns out, it prevents the timelike currents to form altogether.

With the degrees of freedom as obtained in the previous section, we can rewrite Eq. (15) as

$$
\sigma^{\prime \prime}+\frac{1}{r} \sigma^{\prime}=\left[P_{\xi}^{2}+\frac{(Q-n)^{2}}{r^{2}}+\lambda\left(\varphi^{2}+\sigma^{2}-\eta^{2}\right)\right] \sigma,
$$

where now $P_{\xi}^{2}=w(b+e P)^{2}$. In the asymptotic regime, one is left with

$$
\sigma^{\prime \prime}+\frac{1}{r} \sigma^{\prime} \sim\left(w b^{2}+\frac{n^{2}}{r^{2}}\right) \sigma,
$$

as $\sigma$ decreases to vanishingly small values. The general solution for this Bessel equation is

$$
\sigma \sim a_{\mathrm{I}} I_{n}(b \sqrt{w} r)+a_{\mathrm{K}} K_{n}(b \sqrt{w} r)
$$

for constant $a_{\mathrm{I}}$ and $a_{\mathrm{K}}$, with $I_{n}$ and $K_{n}$ the modified Bessel functions of order $n$. For $w>0$, the field is a condensate provided we set $a_{\mathrm{I}}=0$.
The energy contained in this solution converges exponentially fast far from the string core provided $w>0$ : for $w<0$, instead the general solution is a combination of oscillatory Bessel functions. In the usual Witten currentcarrying case [27, 33], there is a similar transition for a given, nonzero negative value $w_{\text {th }}$ of $w$ that leads to a logarithmic divergence in the equation of state in the limit $w \rightarrow w_{\text {th }}$. Here however, the threshold would be for a lightlike current, with $w_{\text {th }}=0$ : the would-be divergence is regularized by the $w$ prefactor that enters into the definition of the energy per unit length and tension (see next Sec. IV and the result is perfectly finite: there is no phase frequency threshold ${ }^{1}$ in this case, the current can, from a spacelike configuration, smoothly evolve towards an almost lightlike situation.

In fact, Eq. (22) also gives the behavior of $\sigma$ with $w$ in the limit $w \rightarrow 0$. First, setting $w=0$ into Eq. 21p yields $\sigma \sim A r^{-n}+B r^{n}$, with $A$ and $B$ unknown constants; a necessary condition for the condensate to be localized on the vortex is that $B=0$. On the other hand, taking directly the solution (22) with $a_{\mathrm{I}}=0$ and expanding the Bessel function $K_{n}$ in the neighborhood of $w \sim 0$ (we assume an analytic continuation with $n \rightarrow n+\epsilon$ and take afterwards the limit $\epsilon \rightarrow 0$ to handle the singularity), one obtains

$$
K_{n}(b \sqrt{w} r) \sim 2^{-1-n} b^{n} r^{n} w^{n / 2} \Gamma(-n)+2^{-1+n} b^{-n} r^{-n} w^{-n / 2} \Gamma(n),
$$

so that, providing $w^{n / 2}$ converges to zero faster than the pole in the $\Gamma$ function, one can identify

$$
a_{\mathrm{K}}=\frac{(b \sqrt{w})^{n}}{2 n-1(n-1) !} A,
$$

where $A$, although arbitrary at this stage, is independent of $w$ as it comes from the solution for $w=0$. Therefore, in the small (but finite) $w$ limit, we have that $\sigma \propto w^{n / 2} K_{n}(b \sqrt{w} r)$ whose asymptotic behavior gives $\sigma \propto w^{n / 2-1 / 4} \mathrm{e}^{-b \sqrt{w} r} / \sqrt{r}$. It is this behavior that implies the chiral current limit to be well defined.

We now move on to evaluating the integrated quantities leading to this equation of state.

\section{INTEGRATED QUANTITIES}

In order to describe the network of strings that will be generated by the single strings here considered, one needs to integrate over the transverse directions in order

\footnotetext{
${ }^{1}$ Rather, one could say that there is a frequency threshold as in the usual case, but the asymptotic mass of the current-carrier vanishes since it is akin to a Goldstone mode here, so the threshold does not imply a divergent behavior of either the energy per unit length or the tension.
} 
to be able to approximate each defect by means of an actually zero thickness object. This means we should derive the current and stress energy tensor associated with the solutions obtained above. As we will then show the string to be unstable with respect to longitudinal perturbations, the worldsheet these integrated quantities suppose will not actually last; assuming its presence is however necessary for calculation purposes.

\section{A. Current}

Among the integrated quantities of interest, the current, defined as

$$
J^{\mu} \equiv \frac{1}{2 e} \frac{\delta \mathcal{L}}{\delta A_{\mu}},
$$

provides two independent ways to verify that the following configurations obtained numerically are indeed solutions and not mere artifacts. With the framework of model (1), this is

$$
J^{\mu}=-\frac{i}{2}\left[\boldsymbol{\Phi}^{\dagger} \cdot\left(\partial^{\mu} \boldsymbol{\Phi}\right)-\left(\partial^{\mu} \boldsymbol{\Phi}^{\dagger}\right) \cdot \boldsymbol{\Phi}\right]-e A^{\mu} \boldsymbol{\Phi}^{\dagger} \cdot \mathbf{\Phi},
$$

which gives, using the explicit form 11 in terms of the components of $\boldsymbol{\Phi}$

$$
J_{r}=0 \quad \text { and } \quad J_{\theta}=Q \varphi^{2}+(Q-n) \sigma^{2},
$$

for the transverse components, and

$$
J_{a}=\sigma^{2}\left(\partial_{a} \xi-e A_{a}\right)-2 e \varphi^{2} A_{a},
$$

with $a \in\{z, t\}$ for the longitudinal, worldsheet components.

Integration over the transverse degrees of freedom yield two macroscopically defined quantities, namely the rotational current flux around the string

$I_{\theta} \equiv \int \mathrm{d}^{2} x^{\perp} J_{\theta}=2 \pi \int\left[Q \varphi^{2}+(Q-n) \sigma^{2}\right] r \mathrm{~d} r=\frac{2 \pi n}{e^{2}}$,

when the field equation (13) with $m=0$ is used, and the Lorentz-invariant current scalar $J$ along the worldsheet defined through

$$
J^{2} \equiv\left(\int \mathrm{d}^{2} x^{\perp} J_{z}\right)^{2}-\left(\int \mathrm{d}^{2} x^{\perp} J_{t}\right)^{2}
$$

which is readily evaluated in terms of the underlying field solution previously derives as

$$
J=2 \pi \sqrt{w} \int\left[e P \varphi^{2}+(b+e P) \sigma^{2}\right] r \mathrm{~d} r,
$$

because the difference of the integrals is itself a squared integral, as expected for Lorentz symmetry reasons along the string. Making use of the field equation (19) then yields $J=0$, so this definition cannot account for a conserved current along the worldsheet. This stems from the fact that the current is now supported by both components of the doublet, whereas in the usual Witten situation, there is only one field that carries the current.

Although mostly useless for physical purposes, the current components (27) and (29) can be used as a measure of the validity of the numerical calculation: once the fields are calculated, evaluating the integrals should reproduce the analytic results above.

An alternative way to define the current is obtained by recalling that it physically comes from the phase gradient along the string. In other words, what really matters is the current-carrying phase instead of the field itself, so that a suitable worldsheet covariant - but not SU(2) covariant - definition is

$$
\mathcal{J}_{a}=-\frac{1}{2} \eta_{a b} \frac{\delta \mathcal{L}}{\delta \partial_{b} \xi},
$$

where $\eta_{a b} \equiv \operatorname{diag}(-1,1)$ is the internal Minkowski metric in the string. Since the action only depends on the phase gradient and not on the phase itself, this current is automatically conserved. With the definition (30), one can construct an integrated current $I$ which is merely one part of that given in 29), namely one finds, using the same integration procedure as in (28) (with the replacements $J \rightarrow I$ and $J_{a} \rightarrow \mathcal{J}_{a}$ )

$$
I=2 \pi \sqrt{w} \int(b+e P) \sigma^{2} r \mathrm{~d} r .
$$

The nonzero value of this quantity also explains the difference between the spacelike and timelike eigenvalues of the stress energy tensor to which we now turn.

\section{B. Worldsheet stress-energy tensor}

From the Lagrangian (1), one also derives the stress energy tensor

$$
T_{\mu \nu}=-2 \frac{\delta \mathcal{L}}{\delta g^{\mu \nu}}+g_{\mu \nu} \mathcal{L}
$$

leading to the worldsheet components

$$
\begin{aligned}
& T_{t t}=2 e^{2} \varphi^{2} A_{t}^{2}+2 \sigma^{2}\left(\partial_{t} \xi-e A_{t}\right)^{2}+A_{t}^{\prime 2}-\mathcal{L}(\varphi, \sigma, Q, P), \\
& T_{z z}=2 e^{2} \varphi^{2} A_{z}^{2}+2 \sigma^{2}\left(\partial_{z} \xi-e A_{z}\right)^{2}+A_{z}^{\prime 2}+\mathcal{L}(\varphi, \sigma, \underset{(33),}{Q, P),} \\
& T_{z t}=2 e^{2} \varphi^{2} A_{z} A_{t}+2 \sigma^{2}\left(\partial_{z} \xi-e A_{z}\right)\left(\partial_{t}-e A_{t}\right)+A_{z}^{\prime} A_{t}^{\prime},
\end{aligned}
$$

where we have made use of the symmetries discussed in the previous sections, and the Lorentz-invariant part stems from the background Lagrangian 
$\mathcal{L}(\varphi, \sigma, Q, P)=-\varphi^{\prime 2}-\sigma^{\prime 2}-\frac{Q^{2} \varphi^{2}}{r^{2}}-\frac{(Q-n)^{2} \sigma^{2}}{r^{2}}-w\left[e^{2} \varphi^{2} P^{2}+(b+e P)^{2} \sigma^{2}\right]-\frac{1}{2}\left(w P^{\prime 2}+\frac{Q^{\prime 2}}{e^{2} r^{2}}\right)-\frac{\lambda}{2}\left(\varphi^{2}+\sigma^{2}-\eta^{2}\right)^{2}$.

We assume the other components, i.e. in the transverse direction, to vanish once integrated along the radial coordinates for the on-shell solution [35]. Following [36], we write

$$
T_{a b}=\left(\begin{array}{cc}
\mathcal{A}+\mathcal{B} & \mathcal{C} \\
\mathcal{C} & -\mathcal{A}+\mathcal{B}
\end{array}\right)
$$

where $\mathcal{A}=-\mathcal{L}(\varphi, \sigma, Q, P ; w \rightarrow 0)$, i.e. that part of $\mathcal{L}$ of Eq. (36) without the variations along the vortex, and

$$
\begin{aligned}
\mathcal{B}= & \varphi^{2} e^{2}\left(A_{z}^{2}+A_{t}^{2}\right)+\sigma^{2}\left[\left(k-e A_{z}\right)^{2}+\left(\omega+e A_{t}\right)^{2}\right] \\
& +\frac{1}{2}\left(A_{z}^{\prime 2}+A_{t}^{\prime 2}\right),
\end{aligned}
$$

and the non diagonal component reads

$$
C=2 \varphi^{2} e^{2} A_{z} A_{t}-2 \sigma^{2}\left(k-e A_{z}\right)\left(\omega+e A_{t}\right)+A_{z}^{\prime} A_{t}^{\prime} .
$$

Diagonalization of $T_{a b}$ with respect to $\eta_{a b}=\operatorname{diag}(-1,1)$ the two-dimensional Minkowski metric yields the eigenvalues $E_{ \pm}$. Those are

$$
\begin{aligned}
E_{ \pm} & \equiv \mathcal{A} \pm \sqrt{\mathcal{B}^{2}-C^{2}} \\
& =\mathcal{A} \pm w\left[\frac{1}{2} P^{\prime 2}+e^{2} P^{2} \varphi^{2}+(b+e P)^{2} \sigma^{2}\right],
\end{aligned}
$$

from which one derives the energy per unit length $U$ and tension $T$ by integration over the transverse degrees of freedom, namely

$$
U=2 \pi \int E_{+}(r) r \mathrm{~d} r \text { and } T=2 \pi \int E_{-}(r) r \mathrm{~d} r .
$$

Note at this point that since the quantity appearing in the diagonalizing solution Eq. (40) is a perfect square, the integration and diagonalization procedures commute, just as in the case of the current for which 29) could be straightforwardly derived, so the resulting macroscopic quantities are really defined in an unambiguous way.

In order to evaluate the actual behavior of the equation of state relating the energy per unit length and the tension, and in particular the stability of the resulting current-carrying string, we now discuss the numerical solutions.

\section{Numerics}

Solving numerically the system of equations (13), (14), (19) and 20, requires that we cancel out the dimensions of the relevant quantities. Setting $\rho=\sqrt{2} e \eta r$

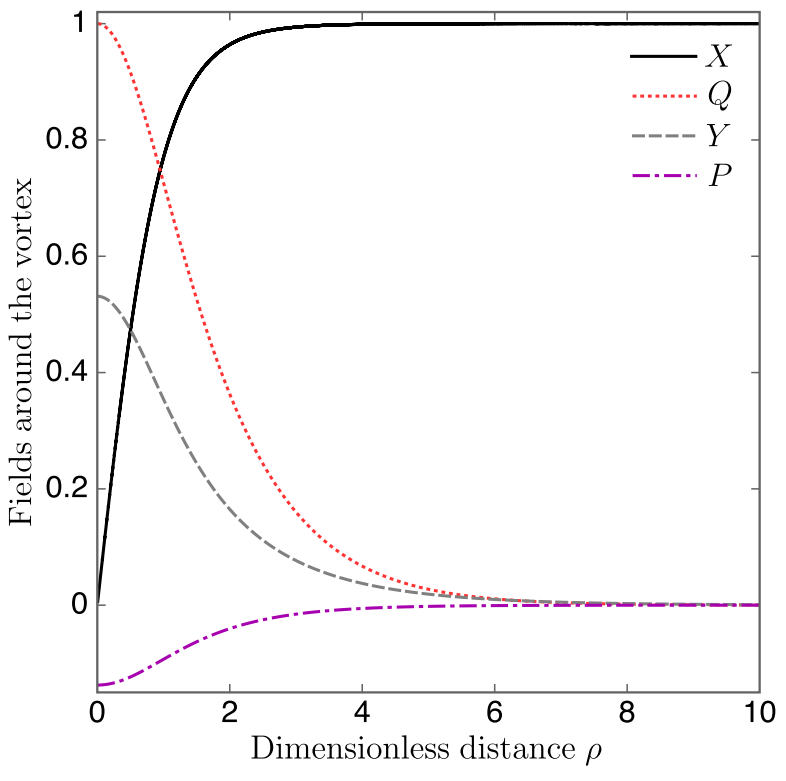

FIG. 2: Rescaled fields around the vortex: $X(\rho)-$ full line and $Y(\rho)-$ dashed - are the Higgs field components in units of the Higgs VEV $\eta$, while the vector field flux $Q(\rho)$ - dotted - renders the vortex local and $P(\rho)$ - dot-dashed - condenses in such a way as to support the current otherwise induced by condensation of $Y$. This figure is obtained for parameter values $\alpha=1, \beta=3$ and $\tilde{w}=0.1 \beta / \alpha^{2}$.

the radius in units of the gauge vector mass, and rescaling the fields and state parameter through

$$
\varphi=\eta X(\rho), \quad \sigma=\eta Y(\rho) \quad \text { and } \quad w=2 \eta^{2} \tilde{w},
$$

we obtain the dimensionless equations of motion in the form

$$
\begin{aligned}
\ddot{X}+\frac{1}{\rho} \dot{X} & =\left[\tilde{w} P^{2}+\frac{Q^{2}}{\rho^{2}}+\beta\left(X^{2}+Y^{2}-1\right)\right] X, \\
\ddot{Q}-\frac{1}{\rho} \dot{Q} & =Q X^{2}+(Q-n) Y^{2}, \\
\ddot{Y}+\frac{1}{\rho} \dot{Y} & =\left[\tilde{w}(\alpha+P)^{2}+\frac{(Q-n)^{2}}{\rho^{2}}+\beta\left(X^{2}+Y^{2}-1\right)\right] Y, \\
\ddot{P}+\frac{1}{\rho} \dot{P} & =P\left(X^{2}+Y^{2}\right)+\alpha Y^{2},
\end{aligned}
$$

where a dot denotes differentiation with respect to the rescaled radius $\rho$ and the constants are defined by $\alpha \equiv$ $b / e$ and $\beta \equiv \lambda /\left(2 e^{2}\right)$. 


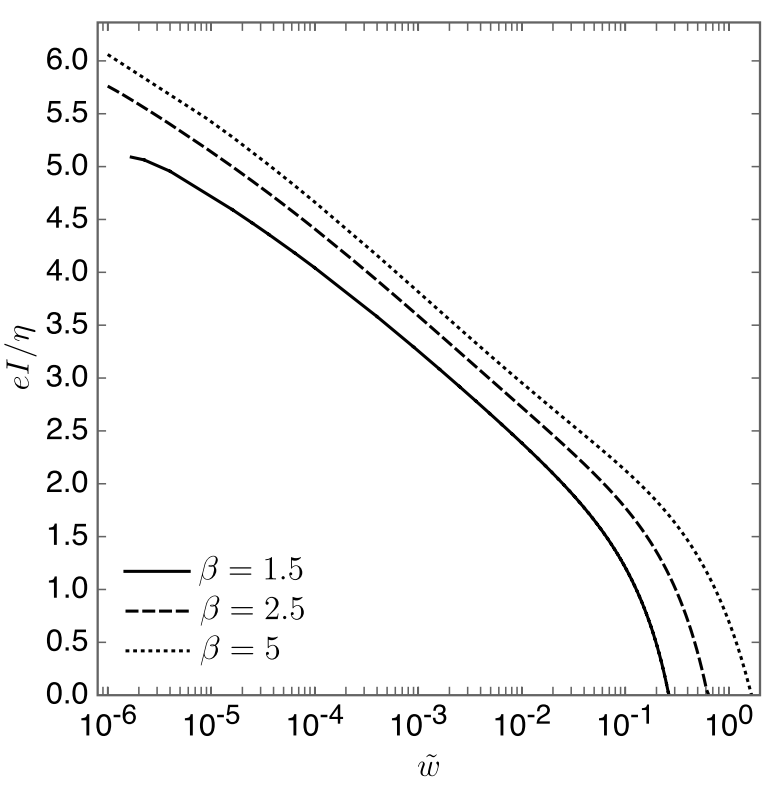

FIG. 3: Variation of the internal current $e I / \eta$ as a function of the rescaled state parameter $\tilde{w}$ for $\alpha=1$ and various values of $\beta$ (same as on Fig. 5).

A point worth discussing in relation with these equations concerns the evolution of the condensate as the state parameter increases. Expanding the field functions around the string core as $X \propto \rho^{m}+\cdots, Y \sim Y_{0}+\frac{1}{2} \ddot{X}_{0} \rho^{2}+$ $\cdots, Q \sim n+\frac{1}{2} \ddot{Q}_{0} \rho^{2}+\cdots$ and $P \sim P_{0}+\frac{1}{2} \ddot{P}_{0} \rho^{2}+\cdots$, where we have taken into account the regular boundary conditions, the zeroth order expansion of Eqs. (42) to (45), one gets that

$$
\ddot{P}_{0}=\frac{Y_{0}^{2}}{2}\left(\alpha+P_{0}\right)
$$

implying that $-\alpha \leq P_{0} \leq 0$ : if $P_{0}>0$, then $(46)$ im- plies that $\ddot{P}_{0}>0$, and hence $P$ should be a growing and positive function of $\rho$, which is inconsistent with the requirement that $\lim _{\rho \rightarrow \infty} P=0$ (we assume, following the figures, that the functions are monotonic). If $P_{0}<-\alpha$, then $\ddot{P}_{0}<0$, the same argument applies with a negative and decreasing function.

Eq. (42) tells us that $m=n$, as usual, while Eq. (43) is trivially satisfied at the lowest order with the given expansion. However, Eq. (44) translates into

$$
\ddot{Y}_{0}=\frac{Y_{0}}{2}\left[\tilde{w}\left(\alpha+P_{0}\right)^{2}+\beta\left(Y_{0}^{2}-1\right)\right],
$$

so that, demanding $\ddot{Y}_{0} Y_{0}<0$ for the reasons just discussed for $P$, one finds that

$$
Y_{0}^{2} \leq 1-\frac{\tilde{w}}{\beta}\left(\alpha+P_{0}\right)^{2}
$$

indicating that for large values of $\tilde{w}$, assuming $P_{0}$ to depend only mildly on $\tilde{w}$ (indeed, $P_{0} \rightarrow 0$ in this limit), the available range for $Y_{0}$ abruptly shrinks to zero when $\tilde{w} \geq \tilde{w}_{\max } \equiv \beta / \alpha^{2}$, or in other words for $w \geq w_{\max } \equiv$ $\lambda \eta^{2} / b$ : the range of variations for the state parameter is automatically constrained, as in the ordinary Witten case [33].

The finite range of variation of the state parameter can be understood in the following way. Imagine a region along the string network where a statistical fluctuation on the phase gradient implies the condensate should form with a very large value of $w$. This gives the would-be condensate enough momentum to pass over the potential barrier (10), and hence blocks the instability to effectively take place until the fluctuation goes to a more reasonable value below the maximum $(\partial \xi)^{2} \leq w_{\max }$.

These equations are derivable from the dimensionless action $\mathcal{S}_{+}$, where

$$
\mathcal{S}_{ \pm}=\int\left\{\dot{X}^{2}+\dot{Y}^{2}+\tilde{w} \dot{P}^{2}+\frac{\dot{Q}^{2}}{\rho^{2}} \pm \tilde{w}\left[X^{2} P^{2}+(\alpha+P)^{2} Y^{2}\right]+\frac{Q^{2} X^{2}+(Q-n)^{2} Y^{2}}{\rho^{2}}+\frac{1}{2} \beta\left(X^{2}+Y^{2}-1\right)^{2}\right\} \rho \mathrm{d} \rho
$$

which is used to produce the numerical solutions shown on Fig. 2 that are discussed below. The quantities $\mathcal{S}_{ \pm}$ serve to define the energy per unit length and tension through

$$
U=2 \pi \eta^{2} \mathcal{S}_{+} \quad \text { and } \quad T=2 \pi \eta^{2} \mathcal{S}_{-} .
$$

We also derive the currents in terms of dimensionless variables as

$$
I=\frac{\eta}{e} \pi \sqrt{2 \tilde{w}} \int(\alpha+P) Y^{2} \rho \mathrm{d} \rho,
$$

It is shown on Fig. 3 as functions of $\tilde{w}$. The limit provided by Eq. (47) compares with our numerical calculations in the sense that the would-be current (50) obtained in Sec. IV A abruptly vanishes when $\tilde{w}$ exceeds the critical value above which the condensate does not 


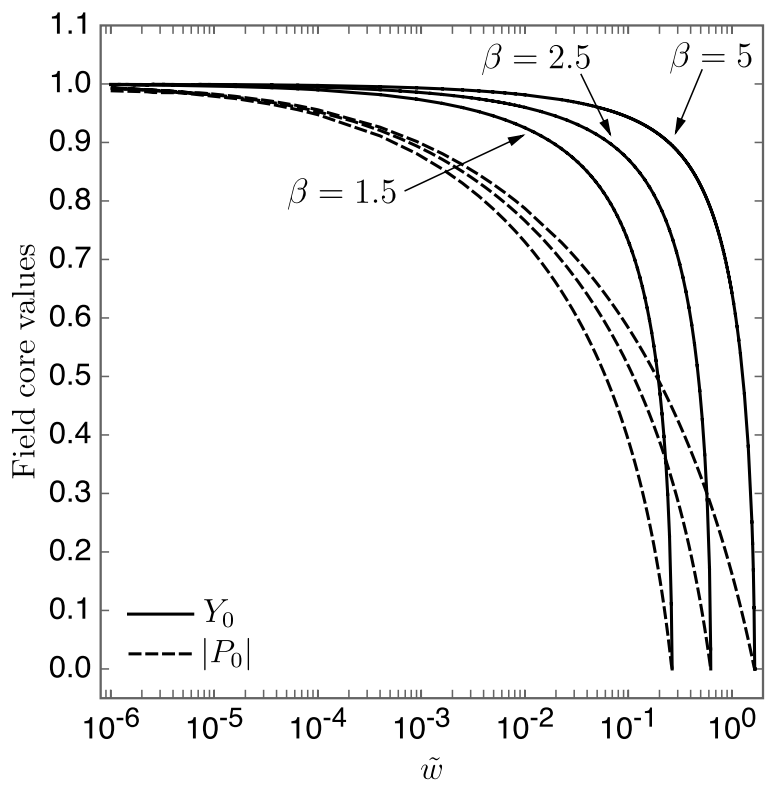

FIG. 4: Values of the condensate function $P_{0}(0)$ and $Y_{0}(0)$ in the string core $(\rho=0)$ as functions of the rescaled state parameter $\tilde{w}$ for $\alpha=1$ and various values of $\beta$ (same as on Fig. 55.

form at all. The other currents, i.e., the constraints stemming from Eqs. 277) and 29], are numerically verified to hold, hence ensuring our field functions to solve their equations of motion.

Eqs. (48) and (49) permit to show explicitely, using the asymptotic behaviors derived above for $\sigma$, that the energy and tension are both well behaved at the wouldbe phase frequency threshold $w \rightarrow 0$. In terms of dimensionless variables, we have, for $\rho \gg 1$, that $Y(\rho)$ behaves as $Y \sim f(\tilde{w}) \tilde{w}^{n / 2-1 / 4} \mathrm{e}^{-\alpha \sqrt{\tilde{w}} \rho} / \sqrt{\alpha \rho}$, where $f(\tilde{w})$ is an unknown function of $\tilde{w}$ whose behavior for small values of the state parameter $\lim _{\tilde{w} \rightarrow 0} f(\tilde{w})$ is a constant.

Now, in this small $w$ regime, it is a simple matter to evaluate the leading behavior of the integrated quantities, as most of the field hardly depend on $w$ : as shown on Fig. 4, the condensate value at the string core and the current gauge function $P$, as well as the background fields $X$ and $Q$, are essentially independent of $w$. The only term that really matters for the variation of the integrals with $w$ is the asymptotic behavior of the current carrier $\sigma$ : as in the ordinary Witten case, the condensate tends to spread around the string around the phase frequency threshold, i.e. here in the almost chiral case. Thus, assuming the asymptotic behavior to hold from a distance $\rho_{\mathrm{M}}$ on, the dominant contribution $\Delta$ comes from the $Y$ terms in Eq. (48), namely

$$
\begin{gathered}
\Delta_{ \pm}=\int_{\rho_{\mathrm{M}}}^{\infty}\left[\dot{Y}^{2} \pm \tilde{w}(\alpha+P)^{2} Y^{2}+\frac{(Q-n)^{2}}{\rho^{2}} Y^{2}\right. \\
\left.+\beta\left(X^{2}-1\right) Y^{2}+\frac{1}{2} \beta Y^{4}\right] \rho \mathrm{d} \rho .
\end{gathered}
$$

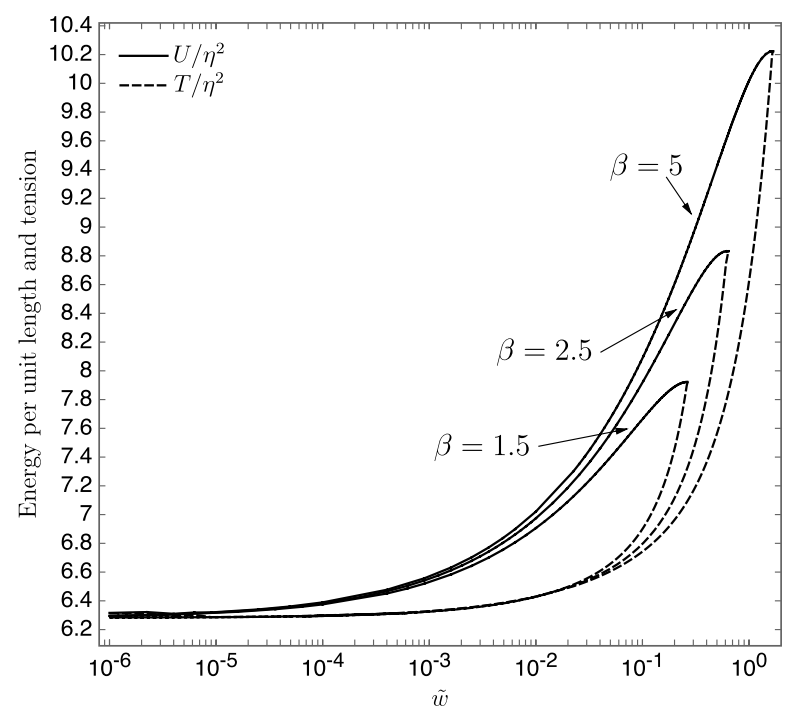

FIG. 5: Energy per unit length $U$ (full lines) and tension $T$ (dashed lines) of the semi-local string in units of the squared Higgs vev $\eta^{2}$ for $\alpha=1$ as on Fig. 2. and different values of $\beta$ as indicated on the curves. This shows explicitly the absence of a phase frequency threshold at $\tilde{w}=0$, i.e. the null current limit is perfectly regular. The functions end abruptly for a maximum value of $\tilde{w}$, as indicated in Eq. (47) after which the condensate identically vanished. It is also seen that $U$ and $T$ vary in the same way with $w$ for all values of $w$, so that $\mathrm{d} T / \mathrm{d} U>0$, and hence the longitudinal perturbation velocity $c_{\mathrm{L}}^{2}$ is always negative, signaling an unstable behavior of the string seen as a macroscopic object; the relevant string evolution presumably leads to a chiral behavior independently of the initial value of the cosmological $w$ distribution at the string network formation time.

For $\rho>\rho_{\mathrm{M}}$, one can further make the assumption that the other fields have reached their asymptotic regime, namely we can set $(P, Q) \rightarrow 0$ and $X \rightarrow 1$, so the only important contributions end up being

$$
\Delta=\int_{\rho_{\mathrm{M}}}^{\infty}\left[\dot{Y}^{2} \pm \tilde{w} \alpha^{2} Y^{2}+\frac{n^{2}}{\rho^{2}} Y^{2}+\frac{1}{2} \beta Y^{4}\right] \rho \mathrm{d} \rho,
$$

which can be explicitly calculated. Neglecting irrelevant constant terms and keeping only the leading contributions, this gives, for $n=1$ (the general case leads to similar conclusions but is merely more involved and, as discussed above, not relevant to the current discussion since the type II vortices here considered are unstable for $n>1$, splitting into $n$ unit winding vortices)

$$
\Delta \sim A \pm B \tilde{w}+C \sqrt{\tilde{w}}+D \tilde{w} \ln \tilde{w},
$$

where $A, B, C$ and $D$ can be evaluated as asymptotic integrals over the fields that do not depend on $\tilde{w}$.

What makes $U$ different from $T$ as functions of $\tilde{w}$ is, in the above expression, the second term involving $B$. In the limit $\tilde{w} \rightarrow 0$, this term rapidly becomes negligible, 
and the dominant contribution thus implies that $U$ and $T$ evolve in similar ways with respect to $\tilde{w}$, the unique parameter describing the string state. As a result, variations of the tension with the energy per unit length are always positive, so the longitudinal perturbation velocity

$$
c_{\mathrm{L}}^{2} \equiv-\frac{\mathrm{d} T}{\mathrm{~d} U} \leq 0
$$

is negative in the limit $\tilde{w} \rightarrow 0$. Numerical calculation shown in Fig. 5f for the full range of available variations of $\tilde{w}$ shows that in fact, Eq. (51) is valid for all possible states attainable by the strings under scrutiny here.

\section{DISCUSSION AND CONCLUSION}

We have investigated a specific model of embedded type II gauged vortices coming from the gauging of a $\mathrm{U}(1)$ subgroup of an otherwise global $\mathrm{SU}(2)$. When the $\mathrm{U}(1)$ symmetry is broken through a Higgs doublet acquiring a nonvanishing vacuum expectation value, another component of the same doublet can be excited because of a well-known condensate instability. This leads to possible current-carrying string states as the phase gradient of the carrier part of the doublet varies along the string: at least at the time when the condensate forms, variations from one point to another are subject to fluctuations over distances larger than the correlation length, i.e. the inverse mass of the Higgs field.

Because type II vortices exhibit only a single unstable mode, it was suggested in [23] that those thus formed could be stable provided they appear as sufficiently small loops so that the unstable long wavelength microscopic perturbations do not take over the dynamics. It remained to understand whether these loops could be macroscopically stable, and this requires that we solve the internal string structure in order to be able to integrate over the irrelevant degrees of freedom. This is achieved by means of a numerical integration of the field equations and a calculation of the relevant integrated quantities forming the stress-energy tensor, to be later coupled to gravity, and the currents. We found that contrary to the original $\mathrm{U}(1) \times \mathrm{U}(1)$ Witten model [7, 33, 34] for which a large region of stability with timelike, lightlike and spacelike currents could be identified, here only a spacelike current could be constructed. This relies on the fact that the condensate is essentially a massless Goldstone mode, so that any timelike excitation would be energetically favored to move away from the string. The lightlike limit, however, appears to be reasonably well-defined.

We obtained another crucial difference with the usual current-carrying string models: the spacelike current configurations happen to be unstable with respect to longitudinal (sound-wave like) perturbations. As a result, our investigation closes the window of possible stability zones opened in Ref. [23], and we are led to the definite conclusion that type II vortices cannot form, or if they do, they will spontaneously decay in such a way that their cosmological relevance is vanishing.

Let us finally point out that the non-current carrying semi-local strings share some features with BPS D-term string solutions [37], in particular the latter possess a zero mode - very similar to semi-local strings. While the zero mode can also be excited [38] one might wonder whether any of the results obtained in our paper would also be valid in this case and whether this could lead to any consequence on inflationary models rooted in String Theory.

\section{Acknowledgments}

BH gratefully acknowledges support within the framework of the DFG Research Training Group 1620 Models of gravity. PP would like to thank support from Jacobs University (Bremen - Germany). This research was supported in part by Perimeter Institute for Theoretical Physics (Waterloo, ON - Canada).
[1] P. Peter and J.-P. Uzan, Primordial cosmology (Oxford University Press, UK, 2009).

[2] R. Jeannerot, J. Rocher, and M. Sakellariadou, Phys. Rev. D 68, 103514 (2003), arXiv:hep-ph/0308134.

[3] S. Clesse, Phys. Rev. D 83, 063518 (2011).

[4] R. Davis and E. Shellard, Phys. Lett. B 209, 485 (1988).

[5] R. Davis and E. P. S. Shellard, Nucl. Phys. B 323, 209 (1989).

[6] R. Brandenberger, B. Carter, A.-C. Davis, and M. Trodden, Phys. Rev. D 54, 6059 (1996), arXiv:hep$\mathrm{ph} / 9605382$.

[7] E. Witten, Nucl. Phys. B249, 557 (1985).

[8] P. Peter, Phys. Rev. D 45, 1091 (1992).

[9] P. Peter, Phys. Rev. D 46, 3335 (1992).
[10] B. Hartmann and B. Carter, Phys. Rev. D 77, 103516 (2008).

[11] T. Vachaspati and A. Achucarro, Phys. Rev. D 44, 3067 (1991).

[12] M. Hindmarsh, Phys. Rev. Lett. 68, 1263 (1992).

[13] M. Hindmarsh, Nucl. Phys. B 392, 461 (1993).

[14] A. Achucarro and T. Vachaspati, Phys. Rep. 327, 347 (2000).

[15] P. Forgács, S. Reuillon, and M. S. Volkov, Physical Review Letters 96, 041601 (2006), arXiv:hep-th/0507246.

[16] P. Forgács, S. Reuillon, and M. S. Volkov, Nuclear Physics B 751, 390 (2006), arXiv:hep-th/0602175.

[17] R. A. Leese, Phys. Rev. D 46, 4677 (1992).

[18] A. Achucarro, K. Kuijken, L. Perivolaropoulos, and 
T. Vachaspati, Nucl. Phys. B 388, 435 (1992).

[19] A. Achucarro, J. Borrill, and A. R. Liddle, Phys. Rev. D 57, 3742 (1998).

[20] A. Achucarro, J. Borrill, and A. R. Liddle, Phys. Rev. Lett. 82, 3742 (1999).

[21] A. Achucarro, P. Salmi, and J. Urrestilla, Phys. Rev. D 75, 121703 (2007).

[22] J. Urrestilla, N. Bevis, M. Hindmarsh, M. Kunz, and A. R. Liddle, JCAP 0807, 010 (2008).

[23] J. Garaud and M. S. Volkov, Nuclear Physics B 799, 430 (2008), 0712.3589.

[24] M. Lilley, F. Di Marco, J. Martin, and P. Peter, Phys. Rev. D 82, 023510 (2010), 1003.4601.

[25] B. Carter, Phys. Rev. D 82, 103531 (2010), 1001.0912.

[26] B. Carter and X. Martin, Ann. Phys. (N. Y.) 227, 151 (1993), arXiv:hep-th/0306111.

[27] B. Carter and P. Peter, Phys. Rev. D 52, R1744 (1995).

[28] B. Carter and P. Peter, Phys. Rev. D 52, R1744 (1995),
arXiv:hep-ph/9411425.

[29] B. Carter and P. Peter, Phys. Lett. B466, 41 (1999), hepth/9905025.

[30] B. Carter and A.-C. Davis, Phys. Rev. D 61, 123501 (2000), arXiv:hep-ph/9910560.

[31] X. Martin and P. Peter, Phys. Rev. D 61, 043510 (2000).

[32] A. Cordero-Cid, X. Martin, and P. Peter, Phys. Rev. D 65, 083522 (2002), arXiv:hep-ph/0201097.

[33] P. Peter, Phys. Rev. D 45, 1091 (1992).

[34] P. Peter, Phys. Rev. D 46, 3335 (1992).

[35] P. Peter, Class. Quant. Grav. 11, 131 (1994).

[36] M. Lilley, P. Peter, and X. Martin, Phys. Rev. D79, 103514 (2009), 0903.4328.

[37] J. J. Blanco-Pillado, G. R. Dvali, and M. Redi, Phys. Rev. D 72, 105002 (2005).

[38] A. Achucarro and K. Sousa, Phys. Rev. D 74, 081701 (2006). 